\title{
44. SHORE LABORATORY REPORT ON THE FORAMINIFERA FROM LEG 27 SITES, DEEP SEA DRILLING PROJECT
}

Richard K. Olsson, Rutgers University, New Brunswick, New Jersey

Tables 1 through 5 show the distribution of planktonic foraminifera at Leg 27 sites, with indication of abundance and preservation. The symbols used in the tables are as follows:

Relative Abundance

$$
0 \text {-Absent }
$$

1-Rare (1-10 specimens)

2-Moderately rare (11-25 specimens)

3-Common (26-50 specimens)

4-Abundant (51-100 specimens)
5-Very abundant (100 specimens)

*-Hundred to thousands of specimens

P-Present

Preservation

D-Dissolution

M-Mechanical erosion

$\mathrm{R}$-Reworked

C-Chalky matrix

$\mathrm{P}$-Poor preservation

$\mathrm{mf}-$ Mixed fauna

TABLE 1

Distribution of Foraminifera, Site 259

\begin{tabular}{|c|c|c|}
\hline Age & Pleistocene & ? \\
\hline Zone & $\begin{array}{l}\text { Globorotalia } \\
\text { truncatu- } \\
\text { linoides } \\
\end{array}$ & ? \\
\hline 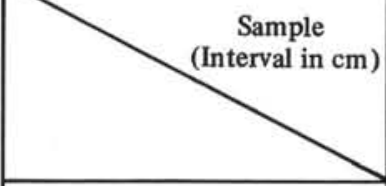 & 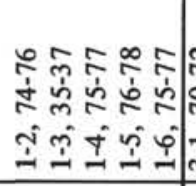 & 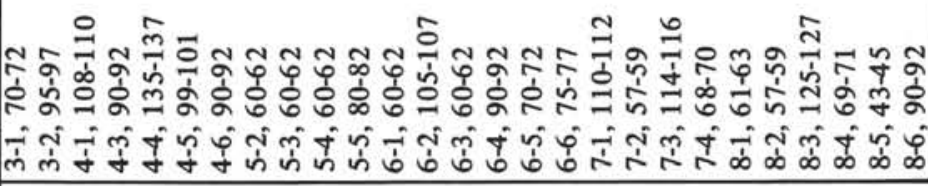 \\
\hline Total Abundance/Preservation & 4D4D 3D 3D 1D & $\begin{array}{lllllllllllllllllllllllllll}0 & 0 & 0 & 0 & 0 & 0 & 0 & 0 & 0 & 0 & 0 & 0 & 1 \mathrm{D} & 0 & 0 & 0 & 0 & 0 & 0 & 0 & 0 & 0 & 0 & 0 & 0 & 0 & 0\end{array}$ \\
\hline $\begin{array}{l}\text { Globorotalia inflata } \\
\text { G. menardii } \\
\text { G. truncatulinoides } \\
\text { G. crassaformis } \\
\text { Globigerina falconensis }\end{array}$ & \begin{tabular}{lllll|}
$*$ & $*$ & 5 & 4 & 1 \\
1 & 1 & 0 & 1 & 0 \\
2 & 1 & 1 & 0 & 0 \\
1 & 0 & 0 & 0 & 0 \\
1 & 0 & 0 & 0 & 0
\end{tabular} & \\
\hline $\begin{array}{l}\text { Globigerinoides trilobus } \\
\text { G. conglobatus } \\
\text { G. ruber } \\
\text { Globigerina dutertrei } \\
\text { Globigerinita glutinata } \\
\end{array}$ & \begin{tabular}{lllll|}
1 & 0 & 0 & 1 & 0 \\
1 & 1 & 0 & 0 & 0 \\
1 & 0 & 0 & 0 & 0 \\
1 & 1 & 1 & 0 & 0 \\
& & & & 1
\end{tabular} & \\
\hline
\end{tabular}


TABLE 2

Distribution of Foraminifera, Site 260

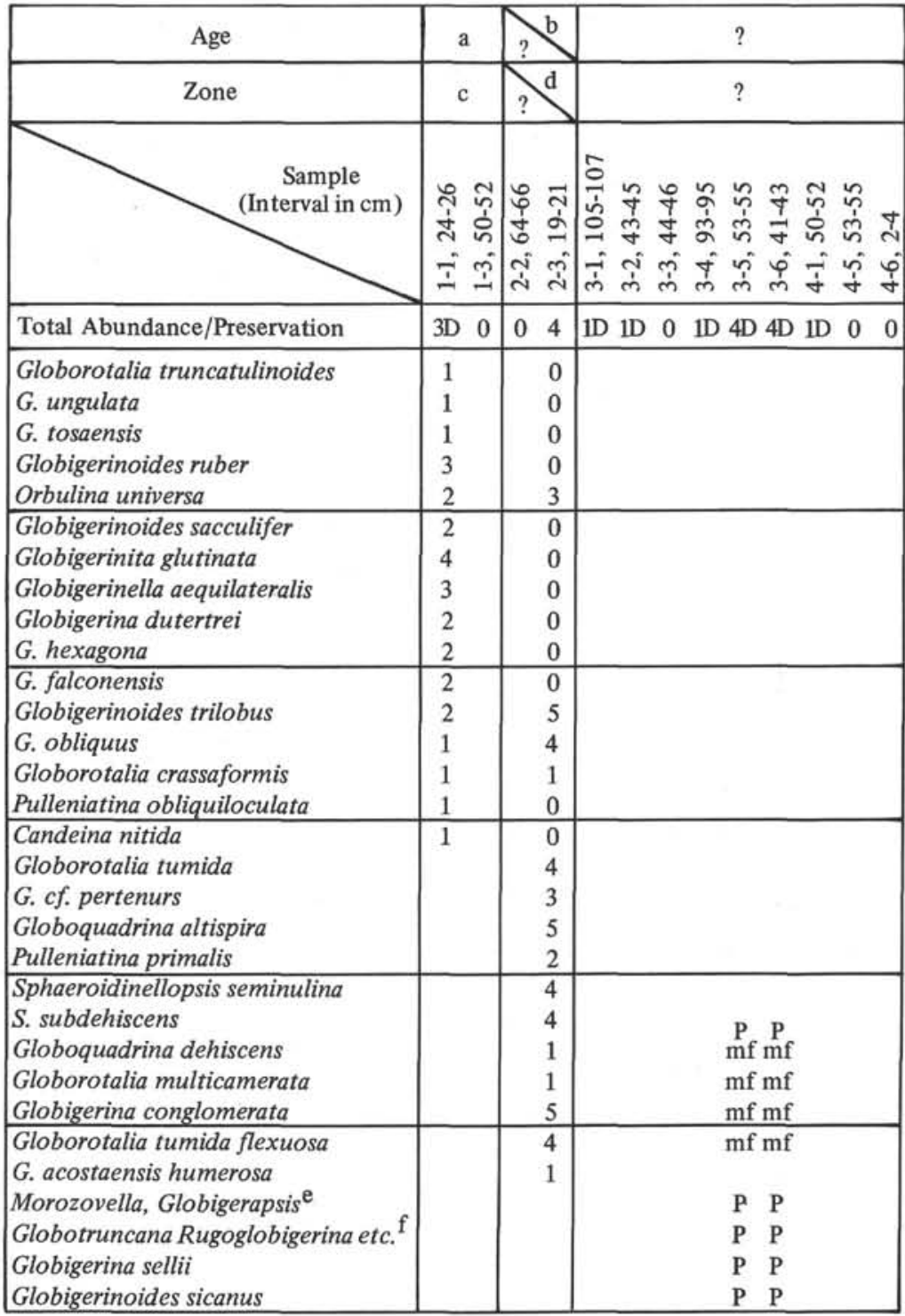

${ }^{\mathrm{a}}$ Pleistocene

${ }^{b}$ Lower Pliocene

c Globorotalia truncatulinoides

${ }^{\mathrm{d}}$ Globorotalia margaritae

${ }^{\mathrm{e}}$ Eocene

${ }^{f}$ Cretaceous

TABLE 3

Distribution of Foraminifera, Site 261

\begin{tabular}{|c|c|}
\hline Age & ? \\
\hline Zone & ? \\
\hline $\begin{array}{c}\text { Sample } \\
(\text { Interval in } \mathrm{cm})\end{array}$ & 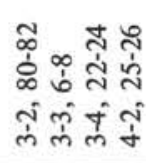 \\
\hline Total Abundance/Preservation & $\begin{array}{llll}0 & 0 & 0 & 0\end{array}$ \\
\hline
\end{tabular}


TABLE 4

Distribution of Foraminifera, Site 262

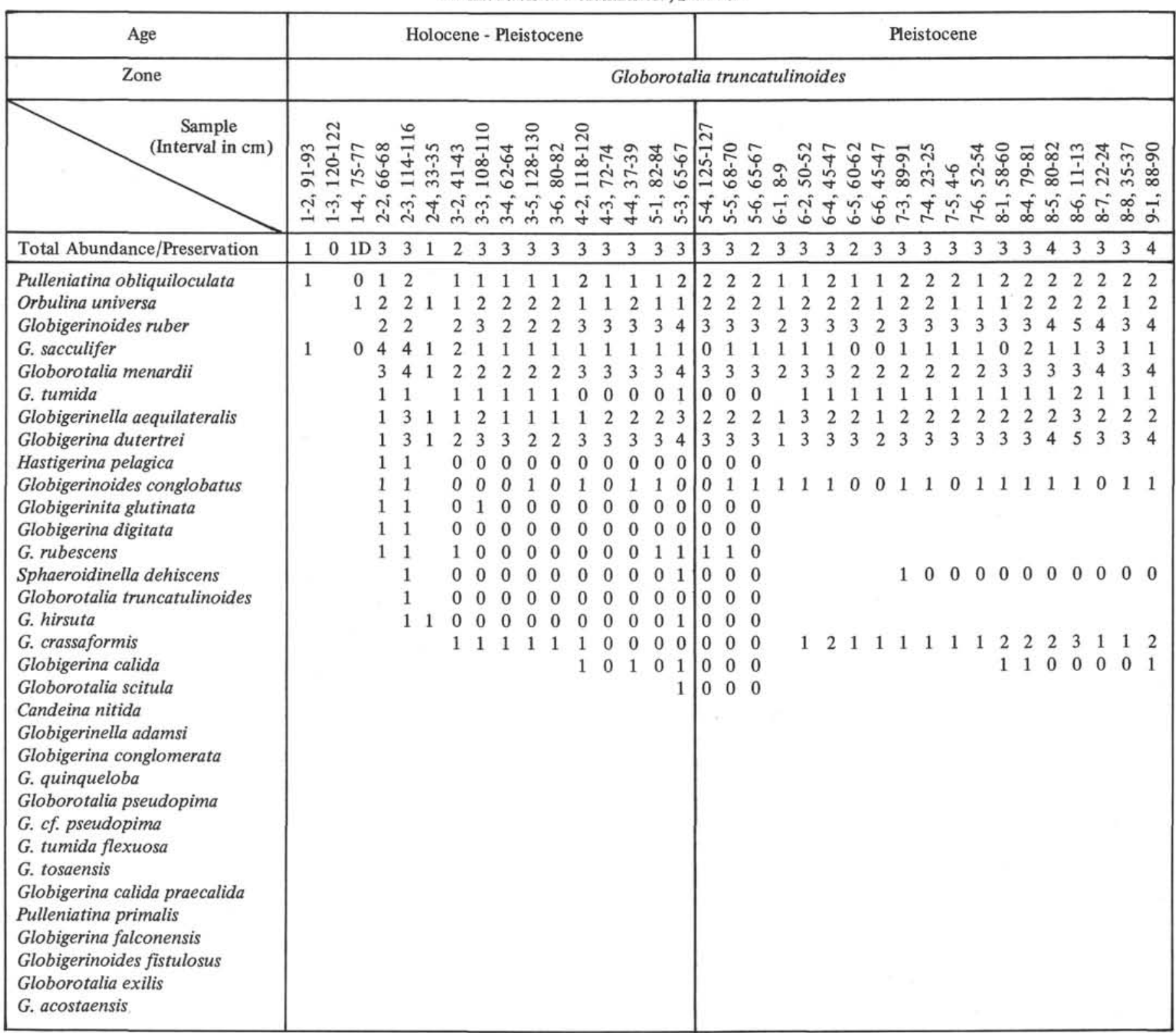




\section{TABLE 4 - Continued}

\section{Pleistocene}

Globorotalia truncatulinoides

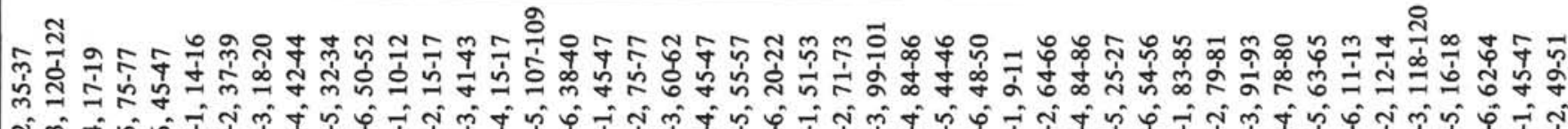

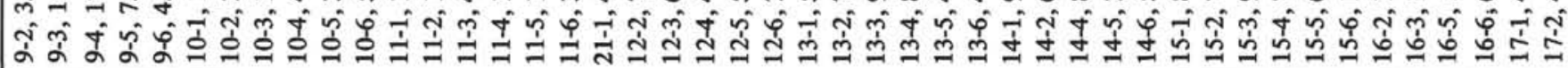

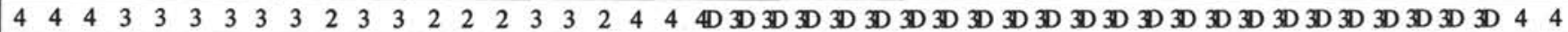

$\begin{array}{lllllllllllllllllllllllllllllllllllllllllllllll}2 & 2 & 2 & 1 & 1 & 1 & 2 & 2 & 2 & 1 & 2 & 2 & 1 & 1 & 2 & 2 & 2 & 1 & 1 & 0 & 4 & 1 & 2 & 2 & 3 & 2 & 3 & 3 & 3 & 1 & 2 & 2 & 3 & 3 & 2 & 3 & 2 & 2 & 3 & 3 & 4 & 4 & 2 & 3 & 3 & 3\end{array}$

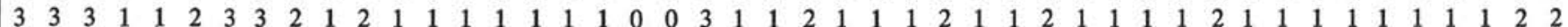
$\begin{array}{lllllllllllllllllllllllllllllllllllllllllllllll}5 & 4 & 4 & 3 & 4 & 4 & 4 & 4 & 4 & 2 & 4 & 4 & 3 & 2 & 3 & 3 & 3 & 3 & 3 & 1 & * & 3 & 2 & 3 & 3 & 3 & 3 & 4 & 3 & 3 & 3 & 2 & 2 & 2 & 2 & 2 & 2 & 4 & 4 & 3 & 3 & 4 & 3 & 2 & 5 & *\end{array}$ $\begin{array}{lllllllllllllllllllllllllllllllllllllllllllllll}1 & 3 & 3 & 2 & 1 & 2 & 1 & 1 & 3 & 1 & 1 & 1 & 1 & 1 & 1 & 1 & 1 & 1 & 1 & 0 & * & 2 & 1 & 1 & 2 & 2 & 2 & 1 & 3 & 3 & 2 & 2 & 2 & 2 & 2 & 2 & 2 & 2 & 2 & 2 & 2 & 1 & 2 & 2 & 2 & 1\end{array}$

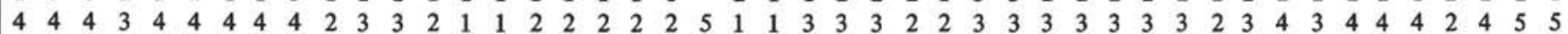
$\begin{array}{llllllllllllllllllllllllllllllllllllllllllllll}1 & 1 & 1 & & 1 & 1 & 1 & 1 & 1 & 1 & 0 & 1 & 1 & 1 & 1 & 1 & 1 & 0 & 0 & 0 & 2 & 1 & 1 & 1 & 1 & 1 & 1 & 1 & 1 & 1 & 1 & 1 & 2 & 2 & 1 & 1 & 2 & 2 & 1 & 2 & 1 & 3 & 1 & 1 & 0 & 0\end{array}$ $\begin{array}{lllllllllllllllllllllllllllllllllllllllllllllll}2 & 3 & 3 & 2 & 3 & 3 & 3 & 3 & 3 & 2 & 3 & 2 & 2 & 1 & 1 & 1 & 1 & 1 & 1 & 1 & 1 & 0 & 1 & 1 & 1 & 1 & 2 & 1 & 1 & 1 & 1 & 1 & 1 & 1 & 1 & 1 & 1 & 1 & 1 & 1 & 2 & 1 & 1 & 1 & 1 & 3\end{array}$ $\begin{array}{lllllllllllllllllllllllllllllllllllllllllllllll}5 & 4 & 4 & 2 & 3 & 3 & 4 & 4 & 4 & 2 & 3 & 3 & 3 & 2 & 3 & 3 & 2 & 2 & 2 & 2 & 5 & 2 & 2 & 3 & 3 & 3 & 3 & 3 & 3 & 3 & 2 & 3 & 3 & 3 & 3 & 2 & 2 & 3 & 2 & 3 & 3 & 3 & 2 & 2 & 4 & 5\end{array}$

$\begin{array}{lllllllllllllllllllllllllllllllllllllllllllllll}1 & 1 & 1 & 1 & 1 & 1 & 1 & 1 & 1 & 1 & 1 & 2 & 1 & 1 & 1 & 0 & 2 & 0 & 0 & 0 & 2 & 0 & 1 & 2 & 1 & 1 & 1 & 2 & 0 & 2 & 2 & 1 & 1 & 1 & 1 & 2 & 1 & 0 & 1 & 1 & 1 & 1 & 1 & 1 & 1 & 3\end{array}$ $\begin{array}{llllllllllllllllllllllllllllllllllllllllllll}1 & 1 & 1 & 1 & 1 & 0 & 0 & 0 & 0 & 0 & 0 & 1 & 0 & 0 & 0 & 5 & * & 3 & 0 & 1 & 4 & 3 & 3 & 2 & 3 & 3 & 2 & 2 & 2 & 3 & 3 & 2 & 2 & 2 & 4 & 4 & 3 & 3 & 4 & 4 & 2 & 2 & 3\end{array}$

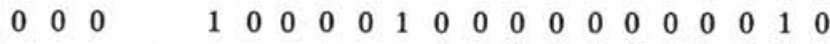
$\begin{array}{lllllllllllllllllllllllllllll}5 & * & 2 & 0 & 2 & 2 & 1 & 1 & 1 & 2 & 1 & 1 & 2 & 1 & 1 & 1 & 1 & 1 & 1 & 1 & 1 & 1 & 1 & 1 & 1 & 1 & 1 & 1\end{array}$ $\begin{array}{lllllllllllllllllllll}1 & 1 & 0 & 0 & 1 & 0 & 1 & 1 & 0 & 0 & 0 & 1 & 1 & 0 & 1 & 1 & & 1 & 0 & 1 & 1\end{array}$ $\begin{array}{lllllllllll}1 & 1 & 0 & 1 & 0 & 0 & 1 & 0 & 1 & 1 & 1\end{array}$ $\begin{array}{lllllllllllllllllllllllllllllllllll}1 & 1 & 1 & 1 & 1 & 1 & 0 & 0 & 0 & 0 & 0 & 1 & 2 & 0 & 0 & 1 & 2\end{array}$

$\begin{array}{lllllllllllllllllllllllllllllllllllllllllllllll}3 & 3 & 3 & 1 & 1 & 1 & 2 & 2 & 1 & 1 & 1 & 1 & 0 & 1 & 1 & 1 & 1 & 1 & 1 & 0 & 1 & 1 & 1 & 2 & 2 & 1 & 1 & 1 & 1 & 2 & 2 & 1 & 1 & 1 & 1 & 1 & 1 & 0 & 0 & 0 & 1 & 1 & 1 & 1 & 2 & 3\end{array}$

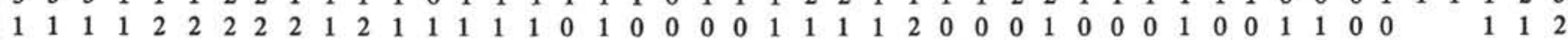
$\begin{array}{llllllllll}1 & 0 & 0 & 0 & 0 & 0 & 0 & 0 & 0\end{array}$ $\begin{array}{llllllllllllll}1 & 1 & 1 & 0 & 1 & 1 & 1 & 1 & 1 & 0 & 1 & 1 & 0 & 0\end{array}$ $\begin{array}{lllllllllllllll}1 & 0 & 0 & 0 & 0 & 0 & 0 & 0 & 0 & 0 & 0 & 0 & 0 & 0 & 0\end{array}$ 
TABLE 4 - Continued

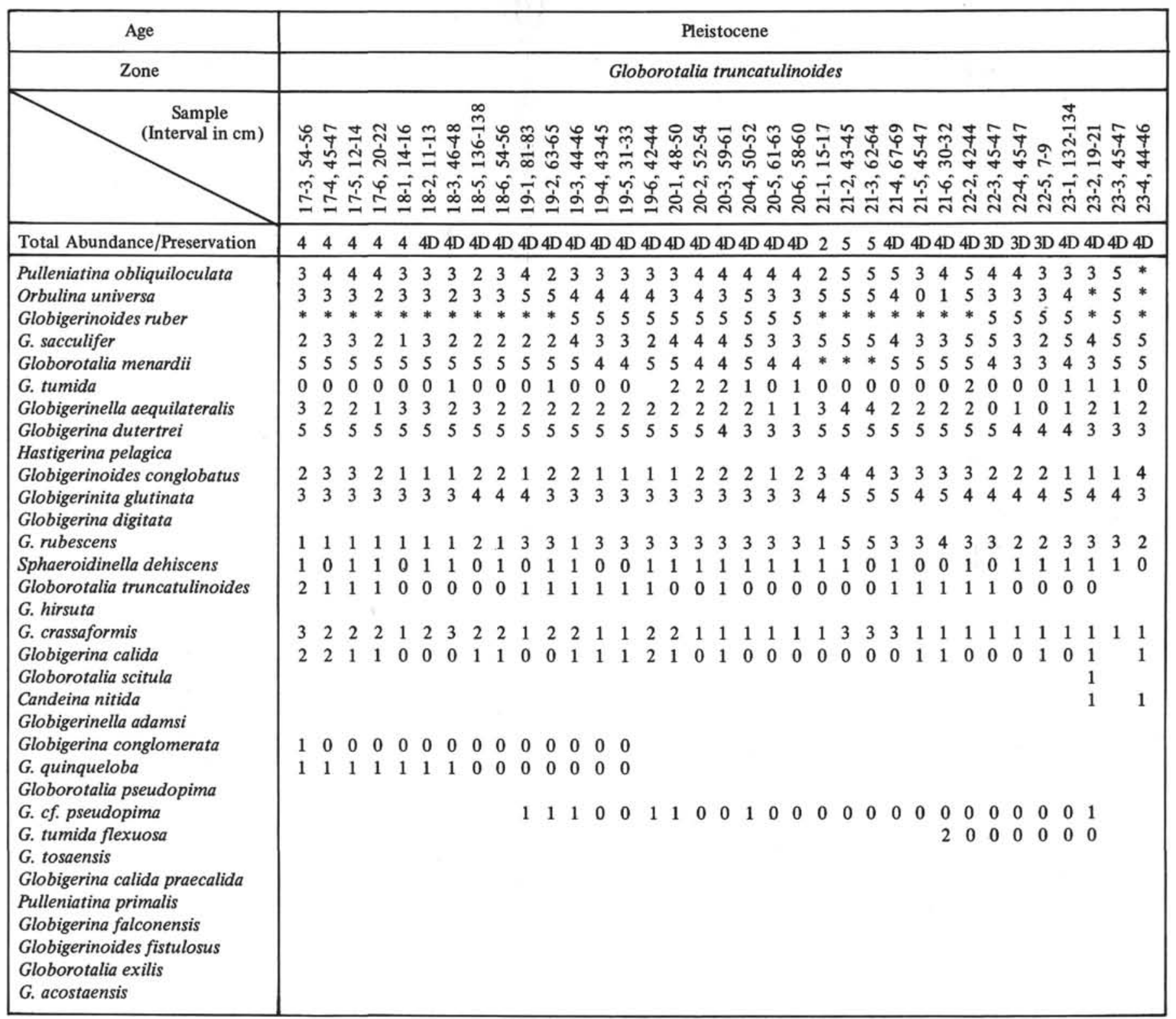


TABLE 4 - Continued

Pleistocene

Globorotalia truncatulinoides

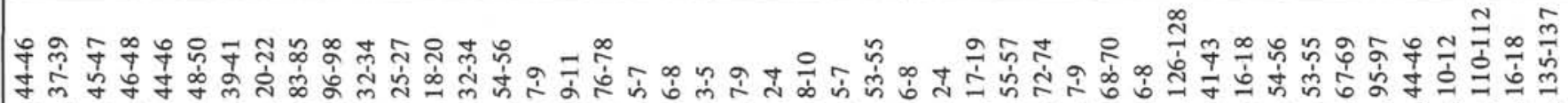

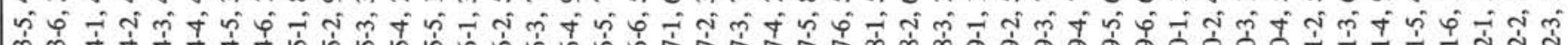

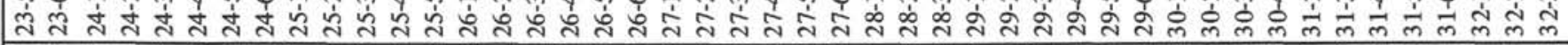
4D 4D 4D 4D 4D 4D 4D 4D 4D 4D 4D 4D 4D 4D 4D 3D 4D 4D 4D 4D 4D 4D 4D 4D 4D 4D 4D 4D 4D 4D 4D 4D 4D 4D 4D 4D 4D 4D 4D 4D 4D 4D 4D 4D 4D 4D

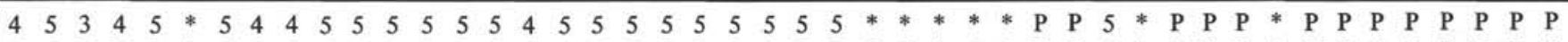

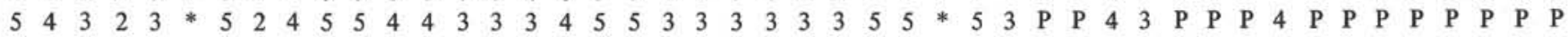

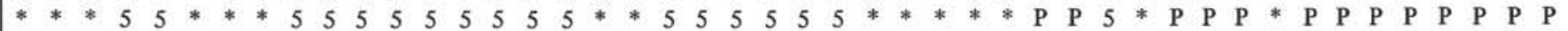

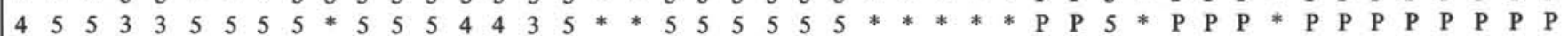

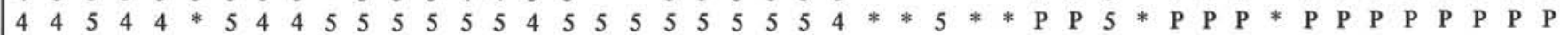

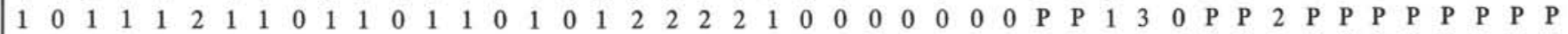

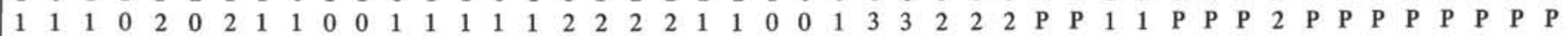

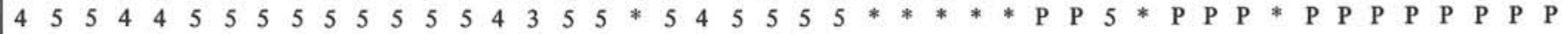

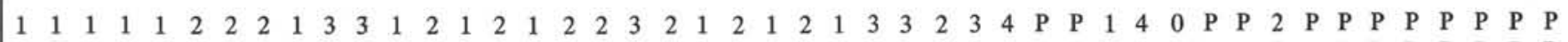

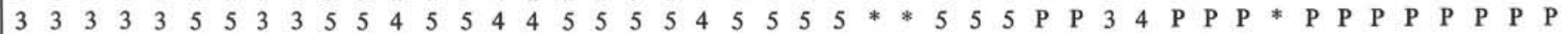

$\begin{array}{llllllllllllllllllllllllllllllllllll}2 & 2 & 2 & 2 & 2 & 3 & 2 & 2 & 2 & 2 & 2 & 2 & 2 & 2 & 2 & 1 & 2 & 2 & 3 & 2 & 1 & 1 & 1 & 1 & 0 & 0 & 0 & 0 & 0 & 0 & 0 & 0 & 0 & 0 & 0 & 0\end{array}$

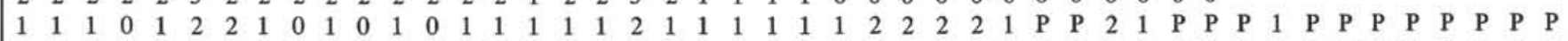

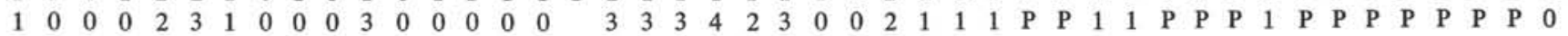

$\begin{array}{lllllllllllllllllllllllllllllllllllll}2 & 1 & 1 & 1 & 1 & 1 & 1 & 0 & 2 & 0 & 1 & 0 & 2 & 2 & 1 & 1 & 1 & 2 & 2 & 2 & 1 & 1 & 0 & 0 & 1 & 2 & 2 & 3 & 3 & 4 & \mathrm{P} & \mathrm{P} & 1 & 0 & 0 & 0\end{array}$ $\begin{array}{llllllllllllllllllllllllllllll}0 & 0 & 1 & 0 & 0 & 0 & 0 & 0 & 0 & 0 & 0 & 0 & 0 & 1 & 0 & 0 & 0\end{array}$

$\begin{array}{lllllllllllllllll}0 & 0 & 0 & 0 & 0 & 0 & 0 & 0 & 0 & 0 & 0 & 0 & 0 & 0 & 0 & 0 & 0\end{array}$ 
TABLE 4 - Continued

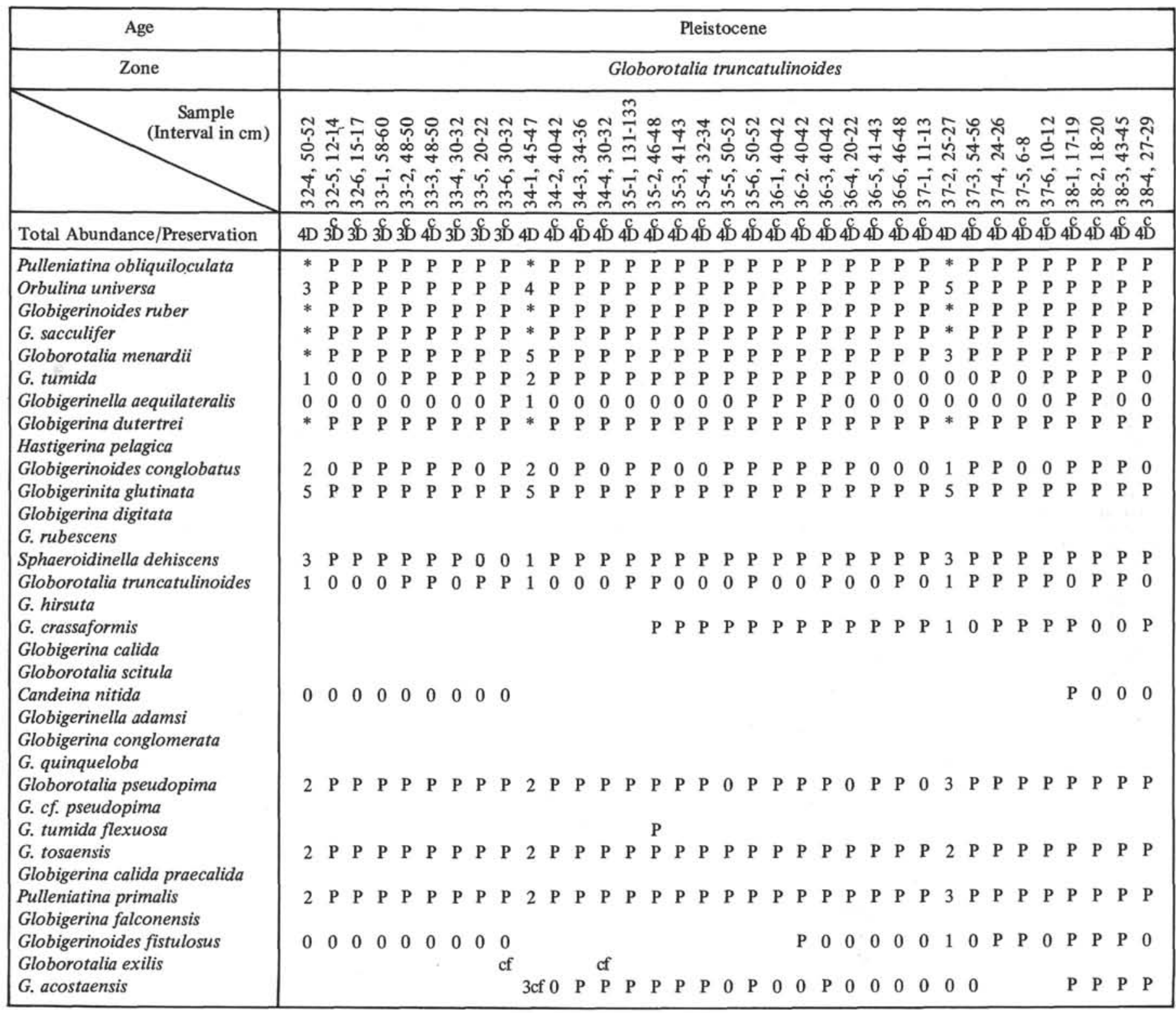


TABLE 4 - Continued

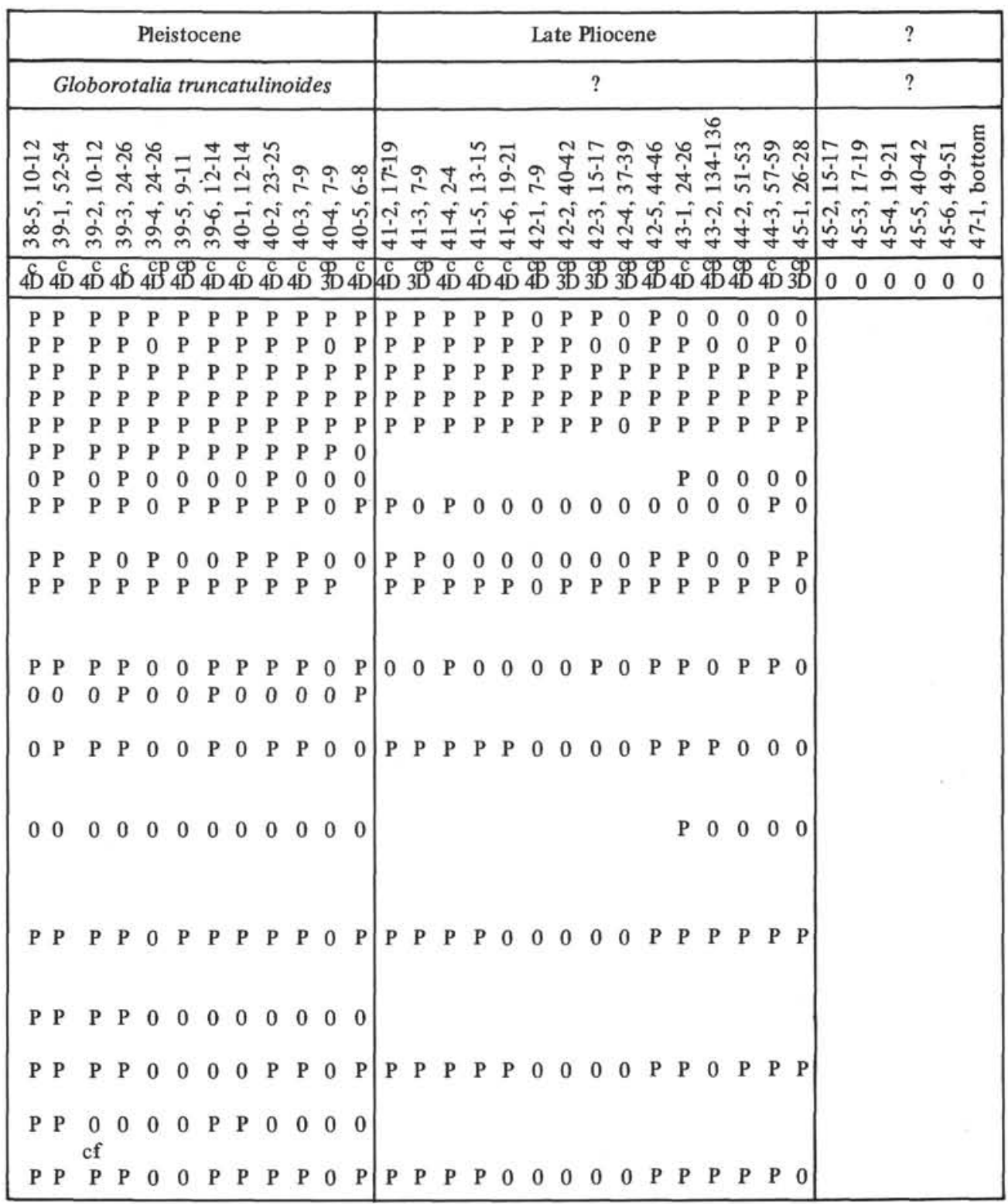


TABLE 5

Distribution of Foraminifera, Site 263

\begin{tabular}{|c|c|c|c|c|c|c|c|}
\hline Age & \multicolumn{5}{|c|}{ Pleistocene } & \multicolumn{2}{|c|}{ a } \\
\hline Zone & & & b & & & c & \\
\hline $\begin{array}{r}\text { Sample } \\
\text { (Interval in }\end{array}$ & $\begin{array}{l}\stackrel{1}{d} \\
\stackrel{1}{1} \\
\stackrel{-}{-}\end{array}$ & $\begin{array}{l}\mathscr{y} \\
\dot{d} \\
\dot{m} \\
\dot{1}\end{array}$ & $\begin{array}{l}\dot{n} \\
\dot{n} \\
\dot{n}\end{array}$ & $\begin{array}{l}\stackrel{\sim}{d} \\
\tilde{d}\end{array}$ & $\begin{array}{l}\bar{\sigma} \\
\text { த் } \\
\dot{ர} \\
\dot{d}\end{array}$ & 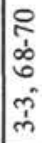 & $\begin{array}{l}\infty \\
\text { yे } \\
\text { dे } \\
\text { के }\end{array}$ \\
\hline Total Abundance/Preservation & 3D & 4 & 4 & 4 & $2 \mathrm{D}$ & $4 \mathrm{D}$ & $4 \mathrm{D}$ \\
\hline Globorotalia truncatulinoides & 2 & 2 & 3 & 2 & 1 & 1 & 1 \\
\hline G. menardii & 1 & 3 & 3 & 3 & 1 & 3 & 3 \\
\hline G. inflata & 3 & * & 4 & 3 & 0 & 0 & 1 \\
\hline G. crassaformis & 2 & 4 & 3 & 2 & 1 & 1 & 3 \\
\hline Globigerina dutertrei & 3 & 4 & 5 & 5 & 1 & 1 & 0 \\
\hline G. hexagona & 1 & 1 & 1 & 1 & 0 & 0 & 0 \\
\hline Globigerinoides ruber & 3 & 5 & * & * & 0 & * & * \\
\hline G. sacculifer & 3 & * & $*$ & $*$ & 1 & * & $*$ \\
\hline G. conglobatus & 1 & 2 & 3 & 3 & 0 & 0 & 2 \\
\hline Globigerinella aequilateralis & 1 & 1 & 1 & 1 & 0 & 1 & 1 \\
\hline Pulleniatina obliquiloculata & 1 & 2 & 3 & 3 & 1 & 1 & 2 \\
\hline Sphaeroidinella dehiscens & & 1 & 1 & 1 & 1 & 0 & 0 \\
\hline Orbulina universa & & 1 & 2 & 3 & 1 & 3 & 4 \\
\hline Globorotalia scitula & & & 1 & 1 & 0 & 1 & 1 \\
\hline G. tumida & & & 3 & 4 & 0 & 0 & 1 \\
\hline Globoquadrina altispira & & & $\mathrm{R}$ & $\mathrm{R}$ & 0 & 2 & 3 \\
\hline Globigerinita glutinata & & 1 & 1 & 1 & 0 & 3 & 2 \\
\hline Sphaeroidinellopsis seminulina & & & & $\mathbf{R}$ & 0 & 0 & 1 \\
\hline Candeina nitida & & & & & & 1 & 1 \\
\hline Globigerina falconensis & & & & & & 3 & 3 \\
\hline Globoquadrina dehiscens & & & & & & 1 & 1 \\
\hline Globotruncana & & & & & & $\mathbf{R}$ & 0 \\
\hline Globorotalia margaritae & & & & & & 1 & 1 \\
\hline Globigerinoides obliquus & & & & & & 3 & 2 \\
\hline Globorotalia cf. multicamerata & & & & & & 1 & 1 \\
\hline Globigerina bulloides & & & & & & & 0 \\
\hline Globigerinoides fistulosus & & & & & & & 1 \\
\hline Globorotalia humerosa & & & & & & & 1 \\
\hline G. $c f$. pertenuis & & & & & & & 1 \\
\hline
\end{tabular}

${ }^{\mathrm{a}}$ Pleistocene/Pliocene ?

${ }^{\mathrm{b}}$ Globorotalia truncatulinoides

$\mathrm{c}_{\text {Mixed Zonal Species }}$ 